\title{
Evidence against a charge density wave on $\mathrm{Bi}(111)$
}

\author{
T. K. Kim, ${ }^{1}$ J. Wells, ${ }^{1}$ C. Kirkegaard, ${ }^{1}$ Z. Li,${ }^{1}$ S. V. Hoffmann, ${ }^{1}$ J. E. Gayone,${ }^{2}$ I. Fernandez-Torrente,${ }^{3}$ P. Häberle,${ }^{3}$ \\ J. I. Pascual, ${ }^{3}$ K. T. Moore, ${ }^{4}$ A. J. Schwartz, ${ }^{4}$ H. He, ${ }^{5}$ J. C. H. Spence, ${ }^{5}$ K. H. Downing, ${ }^{6}$ S. Lazar, ${ }^{7}$ F. D. Tichelaar, ${ }^{7}$ \\ S. V. Borisenko, ${ }^{8}$ M. Knupfer, ${ }^{8}$ and Ph. Hofmann ${ }^{1, *}$ \\ ${ }^{1}$ Institute for Storage Ring Facilities, University of Aarhus, 8000 Aarhus C, Denmark \\ ${ }^{2}$ Institute for Storage Ring Facilities, University of Aarhus, 8000 Aarhus C, Denmark and Centro Atomico Bariloche and CONICET, \\ 8400 S.C. de Bariloche, Argentina \\ ${ }^{3}$ Institut für Experimentalphysik, Freie Universität Berlin, 14195 Berlin, Germany \\ ${ }^{4}$ Chemistry and Materials Science Directorate, Lawrence Livermore National Laboratory, Livermore, California 94550, USA \\ ${ }^{5}$ Department of Physics, Arizona State University, Tempe, Arizona 85287, USA \\ ${ }^{6}$ Lawrence Berkeley National Laboratory, Berkeley, California 94720, USA \\ ${ }^{7}$ National Center for High Resolution Electron Microscopy, Delft University of Technology, Rotterdamseweg 137, 2628 AL Delft, \\ The Netherlands \\ ${ }^{8}$ Institute for Solid State Research, IFW-Dresden, P.O. Box 270016, D-01171 Dresden, Germany \\ (Received 26 May 2004; revised manuscript received 28 March 2005; published 18 August 2005)
}

\begin{abstract}
The Bi(111) surface was studied by scanning tunneling microscopy (STM), transmission electron microscopy (TEM), and angle-resolved photoemission spectroscopy (ARPES) in order to verify the existence of a recently proposed surface charge-density wave (CDW) [Ch. R. Ast and H. Höchst, Phys. Rev. Lett. 90, 016403 (2003)]. The STM and TEM results do not support a CDW scenario at low temperatures. Furthermore, the quasiparticle interference pattern observed in STM confirms the spin-orbit split character of the surface states which prevents the formation of a CDW, even in the case of good nesting. The dispersion of the electronic states observed with ARPES agrees well with earlier findings. In particular, the Fermi contour of the electron pocket at the center of the surface Brillouin zone is found to have a hexagonal shape. However, no gap opening or other signatures of a CDW phase transition can be found in the temperature-dependent data.
\end{abstract}

DOI: 10.1103/PhysRevB.72.085440

\section{INTRODUCTION}

Electronic surface states can be viewed as quasi-twodimensional systems, and as such constitute important test cases for understanding the physics in low dimensions. The increasing tendencies for instabilities such as charge-density waves (CDWs) when going from three-dimensional to lower-dimensional systems is of particular interest. ${ }^{1}$ In fact, there have been several cases of CDWs reported on metal surfaces in recent years, ${ }^{2,3}$ and even typical semiconductor surface reconstructions which turn the surface from a metal into an insulator can be viewed as CDWs. ${ }^{4} \mathrm{~A}$ key ingredient in the formation of a CDW is a singularity in the Lindhard susceptibility, which leads to an unstable situation. Such singularities can be created by a so-called "nesting" of the Fermi contour, i.e., by having many states at the Fermi contour separated by the same wave vector $2 \mathbf{k}_{\mathrm{F}}$.

The (110), ${ }^{5}$ (111), ${ }^{6,7}$ and (100) (Ref. 8) surfaces of Bi are particularly good examples of quasi-two-dimensional metals, because they are good metals whereas the semimetallic bulk material is not. Ast and Höchst have recently reported a spectroscopic indication of a charge density wave formation on Bi(111). ${ }^{9}$ Using high-resolution angle-resolved photoemission spectroscopy (ARPES), they have found that the Fermi surface contour of the so-called electron pocket state around $\bar{\Gamma}$ has a hexagonal shape and therefore fulfills the condition of good "nesting" (for details see Sec. II and Fig. 1). They have also found that the leading edge of the spectra taken at the Fermi surface undergoes a discontinuous shift of about
PACS number(s): 79.60.-i, 71.45.Lr, 73.20. $-\mathrm{r}, 73.25 .+\mathrm{i}$

$5 \mathrm{meV}$ as the sample temperature is lowered from $75 \mathrm{~K}$ to $50 \mathrm{~K}$. This was interpreted as the opening of a gap, caused by the formation of a CDW. However, it was also pointed out that the mere existence of a leading-edge gap would not necessarily imply a CDW ground state. Other techniques are needed to confirm this.

The most remarkable fact about such a CDW on $\mathrm{Bi}(111)$ is that it should not be permitted because of the role of the spin in the electronic structure. It was recently shown that the surface electronic structure of several Bi low-index surfaces is governed by a strong spin-orbit splitting. ${ }^{10}$ This splitting leads to a situation where the surface state bands are completely nondegenerate with respect to spin, i.e., there is only one electron per two-dimensional $\mathbf{k}$ point and band, except for certain high symmetry points where the bands are degenerate because of the combination of time-reversal and crystal symmetry. Time-reversal symmetry dictates that for a state with $\mathbf{k}$ and spin $\uparrow$, there must be a degenerate state with $-\mathbf{k}$ and spin $\downarrow$. This means that states on opposite sides of the Fermi contour have opposite spins. It forbids the pairing needed for a CDW formation but it would leave the possibility of a spin-density wave (SDW). 1,10

In addition to the particular situation on $\mathrm{Bi}$ surfaces, the formation of gaps close to the Fermi level and their spectroscopic signature in ARPES is of considerable general interest, in particular in connection to the subject of hightemperature superconductivity. One is typically interested in the symmetry and size of the superconducting gap or, indeed, if there is any gap at all. ${ }^{11}$ The seemingly straightforward interpretation of ARPES data to yield this information is in 
(a)
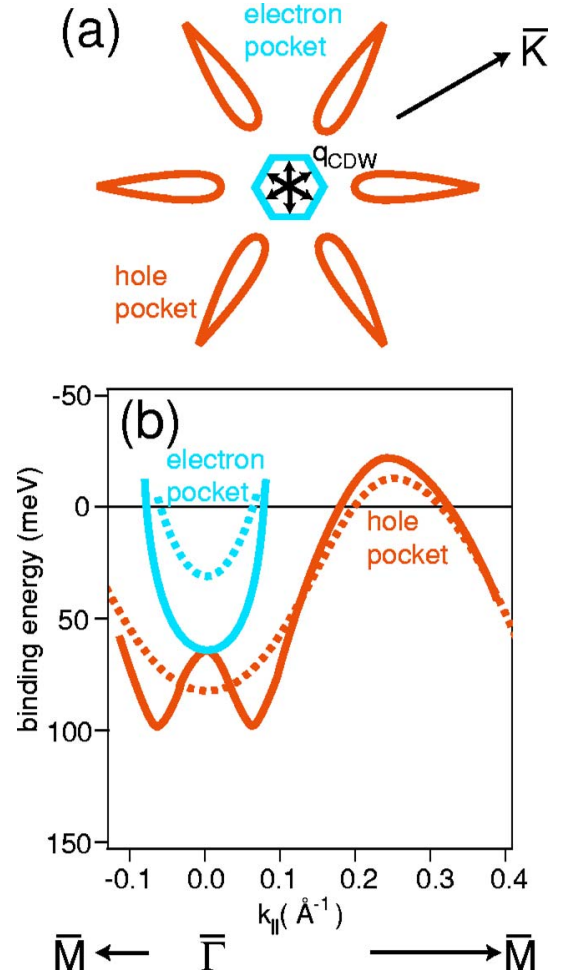

FIG. 1. (Color online) (a) Sketch of the Fermi surface of Bi(111) close to the $\bar{\Gamma}$ point as measured by ARPES (Ref. 7). The black arrows indicate the nesting vector $q_{\mathrm{CDW}}$. (b) Sketch of the electronic band dispersion giving rise to the Fermi surface elements according to the findings of Ast and Höchst (dashed line) (Ref. 7) and Koroteev et al. (solid line) (Ref. 10).

reality not simple; depending on experimental conditions, the size of the inferred gap can have substantial errors and it is even possible to observe apparent gaps for an electronic structure which has no gap. ${ }^{12}$

Here we present a detailed investigation of a possible CDW on $\mathrm{Bi}(111)$. We have used three different techniques to address the issue: scanning tunneling microscopy (STM), transmission electron microscopy (TEM), and ARPES. The former two techniques can give information about the existence of a CDW. STM is the most direct tool because it measures the local density of states (LDOS) close to the Fermi energy. A CDW shows up directly as a LDOS modulation. In addition to this, STM images are very sensitive to the presence of defects such that a locally perfect surface area can be chosen. TEM is also very sensitive to structural modulations and, like STM, it is able to image the real-space structure of the sample such that the sample quality is directly verified. As it turns out, neither STM nor TEM is able to confirm the presence of a surface CDW on $\mathrm{Bi}(111)$. We conclude our investigation by ARPES measurements using two different experimental setups. In neither of these can we confirm the gap opening observed in Ref. 9.

The paper is structured as follows. After this Introduction, we give a brief overview on the electronic structure of $\mathrm{Bi}(111)$. In particular, we focus on the electronic states relevant for a possible CDW formation. In the following three sections we discuss the results from STM, TEM, and
ARPES. In the end, we briefly draw some conclusions.

\section{THE ELECTRONIC STRUCTURE OF BI(111)}

The (111) surface of $\mathrm{Bi}$ has a hexagonal-close-packed structure with a lattice constant of $4.54 \AA$. It is the only surface of rhombohedral Bi crystals which can be created without the breaking of any quasicovalent bonds. ${ }^{13,14}$ Therefore it can be obtained by cleaving single crystals of Bi. After some early ARPES work on $\mathrm{Bi}(111),{ }^{6,15-18}$ a clear picture of the Fermi surface near the center of the surface Brillouin zone (SBZ) was presented by Ast and Höchst. ${ }^{7}$ A simple sketch of their results is shown in Fig. 1(a). There are two Fermi surface elements: a small hexagon around the $\bar{\Gamma}$ point, called the electron pocket, and six lobes around the $\bar{\Gamma}-\bar{M}$ direction, called the hole pockets. A detailed investigation of the electronic state dispersion was presented later by the same authors. ${ }^{19}$

There has been some disagreement about the dispersion of the states at higher binding energies. According to the works of Ast and Höchst, the states forming the electron and hole pockets are not degenerate at $\bar{\Gamma}$ [see Fig. 1(b)]. In an alternative picture, the states have been interpreted as one state split by the spin-orbit interaction. ${ }^{10}$ In this case, symmetry requires the states to be degenerate at $\bar{\Gamma}$. From the experimental data, it is not easy to come up with a definite answer since both states are very broad and weak at $\bar{\Gamma}$, probably due to the interaction with the projected bulk states (see for example Fig. 4 in Ref. 7, Fig. 2 in Ref. 10 or Fig. 5 in the present work). In any event, the precise dispersion of these lower-lying states is of little relevance here.

The electron pocket, i.e., the small hexagon, is the important Fermi surface element for the possible formation of a CDW because of the strong nesting condition which is set up by the parallel Fermi surface lines. ${ }^{9}$ According to Ref. 9, the length of the so-called nesting vector is $2 k_{\mathrm{F}}=q_{\mathrm{CDW}}$ $=0.106 \AA^{-1}$. Such nesting vectors are indicated in Fig. 1(a). Note, that the hexagonal element of the Fermi surface is rotated by $30^{\circ}$ with respect to the hexagonal surface Brillouin zone. This means that the strongest nesting is found in the $\bar{\Gamma}-\bar{K}$ direction in reciprocal space and along the closepacked atomic rows in real space.

As pointed out in the Introduction, an important issue is the spin structure of the electronic states. If the spin-orbit interaction is not an issue, the strong nesting of the electron pocket could easily lead to the formation of a CDW. ${ }^{9}$ If the states are split by the spin-orbit interaction, there cannot be a CDW despite the nesting. Non-spin-resolved photoemission does not give information about the spin. STM does, to some extent, and we will come back to this in Sec. III.

\section{SCANNING TUNNELING MICROSCOPY}

The STM experiments were performed on a single crystal of Bi with a mechanically polished surface. The crystal was cleaned in situ by $\mathrm{Ne}^{+}$sputtering and annealing to about $420 \mathrm{~K}$. Surface cleanliness and structure were checked by STM. The measurements were performed in a low- 

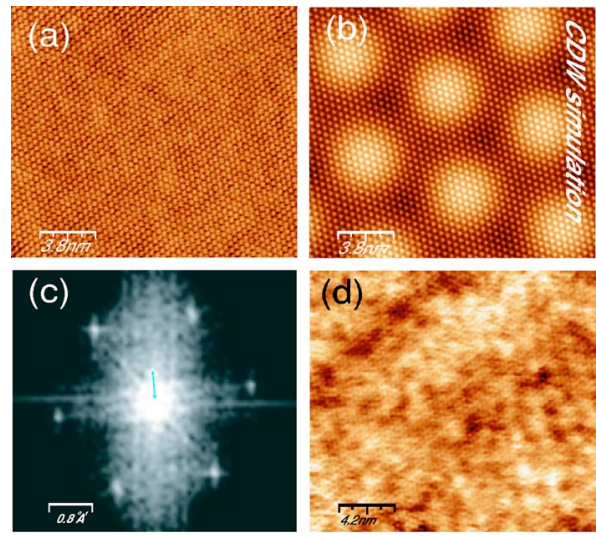

(e)

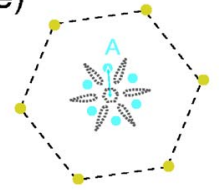

(f)

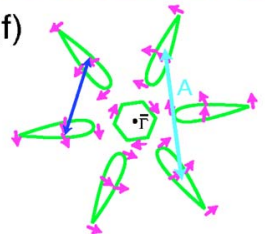

FIG. 2. (Color online) (a) Typical constant current image of $\operatorname{Bi}(111)$ showing the atomic corrugation of the surface $\left(V_{s}=13 \mathrm{mV}, I=1.1 \mathrm{nA}, 19 \times 17 \mathrm{~nm}^{2}\right)$. (b) Model of a CDW modulation superimposed to the $\mathrm{Bi}(111)$ atomic periodicity, in a similar surface area as (a). (d) Differential conductance image $\left(V_{s}=19 \mathrm{mV}, V_{a c}=6 \mathrm{mV} \mathrm{rms}, I=1.1 \mathrm{nA}, 21 \times 19 \mathrm{~nm}^{2}\right)$ and (c) its two-dimensional Fourier transformation. (e) Schematic representation of the Fourier-transformed image. The dashed gray lines are the modulations which would be expected for a spin-independent quasiparticle interference from the Fermi surface in Fig. 1(a). Feature $A$ is the only non-lattice-periodic structure as seen in (c). The yellow markers represent the lattice spots. (f) Illustration of the Fermi surface with the approximate spin direction indicated. The transition giving rise to the $A$ features is indicated. The dark arrow illustrates one of the many possible small- $k$ transitions which do not violate the spin conservation.

temperature STM at a sample temperature of $5 \mathrm{~K}$, far below the transition temperature of the alleged CDW state.

Figure 2(a) shows a STM constant current image taken at a tunneling voltage of $13 \mathrm{mV}$ and a current of $1.1 \mathrm{nA}$. The image clearly shows the atomic corrugation corresponding to the hexagonal lattice of $\mathrm{Bi}(111)$, but no sign of a chargedensity wave. In order to illustrate this, Fig. 2(b) shows a simple model of the $\mathrm{Bi}(111)$ atomic structure with the superimposed modulation of the wave vector $\mathbf{q}_{\mathrm{CDW}}$. The STM image does not resolve such CDW modulation even for tunneling into states close to the Fermi level. This result represents solid evidence against the presence of a CDW on $\mathrm{Bi}(111)$, since CDWs usually show up very clearly in constant current STM images. ${ }^{20-23}$ It is also interesting to note that earlier low-temperature STM investigations of $\mathrm{Bi}(111)$ did not report the observation of a CDW superstructure either, although the precise temperature in these experiments is not known. ${ }^{24,25}$

It has been shown recently for $\mathrm{Bi}(110)$ that the quasiparticle interference patterns observed in STM conductance images can be used to infer information about the spin of the electronic states. ${ }^{26}$ A quasiparticle interference pattern (at the Fermi energy) arises because a quasiparticle with wave vec- tor $\mathbf{k}_{\mathrm{F}}$ is reflected into a state with wave vector $-\mathbf{k}_{\mathrm{F}}$ when it encounters a defect such as an impurity or a step edge. ${ }^{27}$ The interference of incoming and reflected waves gives rise to a modulation in the local density of states with a periodicity of $2 \mathbf{k}_{\mathrm{F}}$, i.e., with the vector connecting the two states. A twodimensional Fourier transformation of conductance images taken with small tunneling voltages is therefore closely related to the two-dimensional Fermi surface, ${ }^{28}$ a relationship which has led to several applications. ${ }^{21,28-35}$ However, this simple picture is only correct when the state at $\mathbf{k}_{\mathrm{F}}$ and the state at $-\mathbf{k}_{\mathrm{F}}$ have the same spin. $\mathrm{On} \operatorname{Bi}(110)$ this is not the case because of spin-orbit splitting. Simple $2 k_{\mathrm{F}}$-type interference patterns can therefore not be observed and the quasiparticle interference patterns are dominated by more complicated, spin-conserving scattering events. ${ }^{26}$

Information about the spin of the states near the Fermi level is, of course, extremely relevant here because of the great similarity between quasiparticle interference and the $2 k_{\mathrm{F}}$ interaction which results in the formation of a CDW. For $\operatorname{Bi}(111)$ surface theory predicts a strong spin-orbit split of the surface states $;{ }^{10}$ states in the electron and hole pocket are not spin degenerate. In fact, if we consider one direction of reciprocal space, states at the hole pocket will present opposite spin orientation than those at the electron pocket. Therefore, we expect that, as for $\mathrm{Bi}(110)$, quasiparticle interference on $\operatorname{Bi}(111)$ only will be present between states which are not completely orthogonal.

Figures 2(d) and 2(c) show a conductance image taken at $19 \mathrm{mV}$ and $1.1 \mathrm{nA}$ and its Fourier transformation (FT), respectively. The latter shows a set of six spots in a hexagonal fashion close to the $k=0$ point (labeled $A$ ), in addition to the points corresponding to the hexagonal atomic lattice. A schematic sketch of the FT map is also given in Fig. 2(e) together with the expected interference contour for a situation in which the spin is not important (dashed gray lines). As on $\mathrm{Bi}(110)$, interference between states with opposite wave vectors is not observed. The only interference which leads to distinct extra features in the Fourier-transformed image is the set of spots labeled $A$. Note that interference events between states with $\mathbf{q}_{\mathrm{CDW}}$ could probably not be identified easily in the Fourier-transformed image because $\mathbf{q}_{\mathrm{CDW}}$ is so small (about four times smaller than the wave vector pointing to $A$ ) that the structures would be very close to the high intensity at the center of the Fourier-transformed image. However, a pronounced interference, as could be expected from the strong nesting, would probably show up in the real-space conductance image in a similar way as in Fig. 2(b). Scattering events between hole pocket states with $\mathbf{k}_{\mathrm{F}}$ and hole pocket states with $-\mathbf{k}_{\mathrm{F}}$, on the other hand, should be easily identifiable and are clearly not observed. We can take this as direct evidence for the spin-orbit split character of the surface states predicted by theory. ${ }^{10}$

The $A$ features are interpreted as transitions between the hole pocket lobes as indicated in Fig. 2(f). The spin of the states involved in such transitions is not exactly parallel but it is not orthogonal either, leading to a finite transition probability. In addition to these events, many spin-conserving small- $k$ scattering events can be expected to contribute to the weak waves observed in Fig. 2(d). One of these events is indicated by the dark arrow in Fig. 2(f). Such transitions are 
expected to contribute to the high intensity of the Fouriertransformed image around the origin.

The main conclusions from this section are that no charge-density wave is observed in low-temperature scanning tunneling microscopy on $\mathrm{Bi}(111)$ and that Fouriertransformed conductance images confirm the spin-orbit split character of the surface states forming the Fermi surface.

\section{TRANSMISSION ELECTRON MICROSCOPY}

Transmission electron microscope experiments were performed using three separate microscopes: A Philips CM300 field-emission gun, a JEOL JEM-3100-FEF field-emission gun, and a Philips CM30T. Measurements could be taken at three different temperatures: $\approx 300 \mathrm{~K}, \approx 90 \mathrm{~K}$ when cooling with liquid nitrogen, and $\approx 18 \mathrm{~K}$ when cooling with liquid helium. Samples of $99.99 \%$ bismuth were prepared by dimpling and ion milling to electron transparency and examined via bright-field imaging and electron diffraction in the transmission electron microscope. A conservative estimate of the smallest sample thickness investigated in this study is 20(5) $\mathrm{nm}$.

We have examined diffraction patterns assuming the suggested incommensurate surface $\mathrm{CDW}$ with $q_{\mathrm{CDW}}$ $=0.106 \AA^{-1}$. The expected location of the CDW reflections is shown in Fig. 3(a) by red markers around the $-1,1,0$ Bragg reflection. The zone axes shown are 111, as indexed in Fig. 3(b). The CDW spots would appear as satellite spots around each of the main Bragg reflections of Bi in a form of $m \mathbf{q}_{\mathrm{CDW}}$ $(m= \pm 1, \pm 2, \ldots)$ with usually only $m= \pm 1$ visible. The orientation of the CDW superlattice reflections relative to the Bragg spots depends on how the Fermi surface nesting vector is oriented with respect to the Brillouin zone. For clarity, the surface Brillouin zone, the electron pocket Fermi surface, and the nesting vectors are also indicated in Fig. 3(a). As pointed out in Sec. II, the hexagonal electron pocket is rotated by $30^{\circ}$ with respect to the hexagonal surface Brillouin zone such that the nesting vectors are also rotated by $30^{\circ}$ with respect to the reciprocal lattice vectors.

TEM has the unique capability of examining either bulk or surface features of a sample, depending upon experimental parameters. We have therefore first examined the bulk, solely to rule out any possibility of a bulk CDW. The sample was oriented along multiple zone axes and electron diffraction patterns were recorded at room temperature and when cooling with liquid nitrogen or liquid helium. As expected, no superlattice reflections were observed that were indicative of a bulk CDW. At each temperature the principle Bragg reflections were strong and well defined, but no evidence of CDW superlattice reflections appeared with $q_{\mathrm{CDW}}$ near $0.106 \AA^{-1}$. After clarifying this, we have concentrated on the possibility of a surface CDW.

In order to examine the potential for a surface CDW, electron diffraction patterns were recorded at various temperatures with the sample tilted into specific diffracting conditions. Electron diffraction patterns were also recorded from thick to thin parts of the sample, since the contribution of surface effects is increased as the sample thickness is reduced. From our electron diffraction experiments, performed
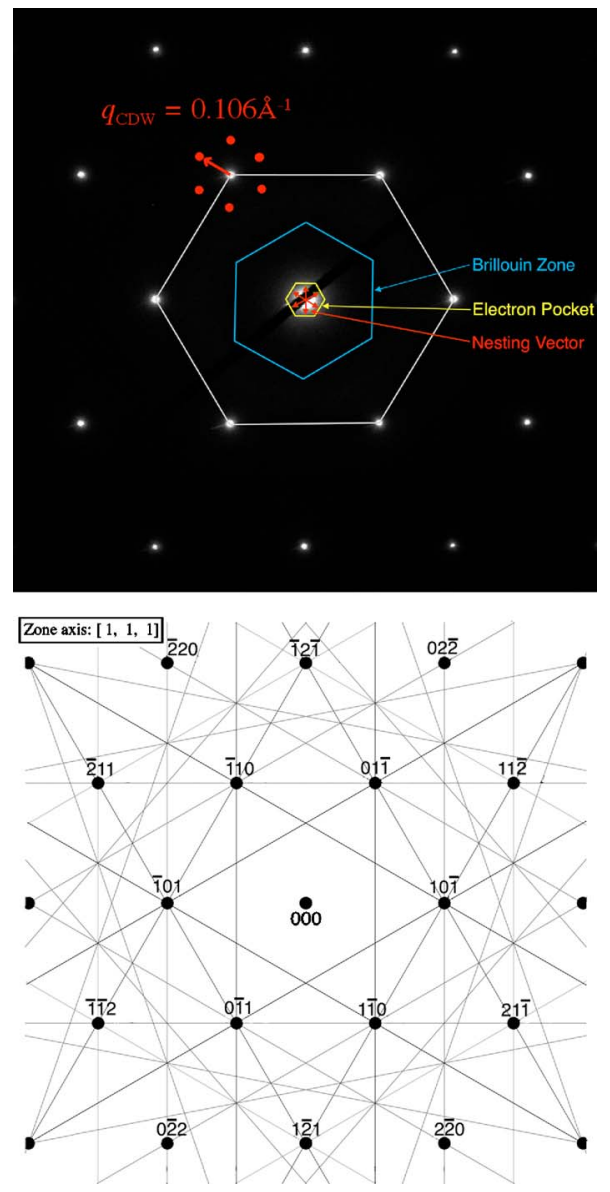

FIG. 3. (Color online) (a) Diffraction image of $\mathrm{Bi}(111)$ as obtained by TEM. The red markers correspond to the expected position of CDW satellite spots. A sketch of the surface Brillouin zone, the hexagonal electron pocket, and the nesting vectors is also given. (b) Indexing of the zone axes.

in two places by two different groups, we found no convincing satellite reflections at or near the positions corresponding to those suggested by Ast and Höchst. ${ }^{9}$

It remains to be proven that reflections due to a surface CDW would be detectable under our experimental conditions. We begin by assuming that there is one CDW layer on the surface of $\mathrm{Bi}$, and the amplitude of atomic displacement in that layer is similar to other CDW systems. Then we ask how thick the specimen can be such that this single-layer CDW is still observable by electron diffraction. If our sample was thin enough to allow observation of a surface CDW state, then our data are relevant.

We first estimate the intensity of the CDW satellite reflection, which depends on the relative thickness of CDW to specimen. We then set up a criterion from a statistical point of view. The estimated intensity of the CDW is approximately

$$
I_{\mathrm{CDW}} \approx 2 \frac{d_{\mathrm{CDW}}}{d_{\mathrm{SP}}} R_{\mathrm{C} 2 \mathrm{~B}} I_{\mathrm{B}},
$$

where $I_{\mathrm{CDW}}$ is the estimated CDW satellite reflection intensity, $d_{\mathrm{CDW}} / d_{\mathrm{SP}}$ is the ratio of the $\mathrm{CDW}$ thickness to specimen 
thickness, the factor of 2 accounts for the two surfaces, $R_{\mathrm{C} 2 \mathrm{~B}}$ is the ratio of intensity of CDW satellite reflection to Bragg peak, and $I_{\mathrm{B}}$ is the intensity of the associated Bragg peak. We have also assumed the intensity from CDW is proportional to thickness (volume), which is true if the integrated intensity is used. The factor $R_{\mathrm{C} 2 \mathrm{~B}}$ cannot be measured. We estimate this empirically, from other typical CDW systems, such as $U .^{36}$ Typically, CDW reflections have an intensity of $10^{-3}$ to $10^{-4}$ times primary Bragg reflections. In our calculations we use the lower limit, assuming the weakest possible CDW reflections.

Next we have to determine the largest possible specimen thickness $d_{\mathrm{SP}}$, for which our signal-to-noise ratio would still allow us to detect a surface CDW. We assume that we have $S$ scattered electrons due to the CDW and $N$ scattered electrons due to inelastic scattering and other processes as a background. The CDW will be observable if it leads to a count rate above the noise of the background, i.e., if $S>2 \sqrt{N}$. As a final condition we get

$$
d_{\mathrm{SP}}<2 \frac{d_{\mathrm{CDW}}}{2 \sqrt{N}} R_{\mathrm{C} 2 \mathrm{~B}} I_{\mathrm{B}}
$$

By assuming a surface CDW layer with a thickness of $2 \AA$ on each surface (top and bottom of the TEM specimen), $R_{\mathrm{C} 2 \mathrm{~B}}$ of $10^{-4}, I_{\mathrm{B}} \approx 10^{7}$ counts, and $N \approx 16$ counts, the above consideration leads to $d_{\mathrm{SP}}<50 \mathrm{~nm}$, which is well above the minimum film thickness studied by us.

The conclusion from our TEM experiments is therefore that the existence of a surface $\mathrm{CDW}$ on $\mathrm{Bi}(111)$ can be excluded.

\section{ANGLE-RESOLVED PHOTOEMISSION}

Having now firmly established that $\mathrm{Bi}(111)$ does not undergo a surface CDW transition, it is interesting to reinvestigate the issue of a leading-edge gap in angle-resolved photoemission at low temperatures. ARPES data were taken at two experimental setups with somewhat complementary qualities.

The first set of data were taken at the SGM-3 beamline of the synchrotron radiation source ASTRID (Aarhus storage ring in Denmark) in Aarhus. ${ }^{37}$ The total energy resolution (of the photon source and the electron analyzer) for the data shown below is $26 \mathrm{meV}$. The angular resolution of the analyzer was set to $\pm 0.2^{\circ}$. The sample could be cooled to approximately $30 \mathrm{~K}$ with a closed-cycle He cryostat. Data were taken on a mechanically polished $\mathrm{Bi}(111)$ crystal which was cleaned in situ by cycles of $\mathrm{Ne}^{+}$sputtering and annealing to $473 \mathrm{~K}$. Surface cleanliness was monitored with Augerelectron-spectroscopy (AES). Surface order was checked by low-energy electron diffraction (LEED). Our Bi(111) crystal showed a good $(1 \times 1)$ LEED pattern.

Measurements at higher temperatures were performed by heating the sample with the radiation from a filament mounted behind it. Heating and data collection were chopped to avoid any influence of the filament's magnetic field on the measurements. The absolute orientation of the sample was determined by ARPES measurements. In the present case this is very simple because of the distinct and narrow Fermi surface features. The relative orientation of the sample for different temperatures was monitored by a laser reflected from the surface onto the wall of the laboratory. In this way we could make sure that the sample orientation changed by less than $0.02^{\circ}$ over the temperature range investigated here. Another possible source of error is a slight movement of the UV light spot over the sample surface during the temperature scans since this can change the apparent emission angle by a small fraction of a degree. We have monitored this by taking fast momentum distribution curves (MDCs) at the Fermi level before and after the temperature scans. The position of the features appeared unchanged.

The other set of data was taken using a Scienta SES200 analyzer and a UV lamp with a monochromator at the Institute for Solid State Research in Dresden. The same sample and the same preparation method was used. This data set is complementary to the data taken in Aarhus. There are differences in the energy resolution $(13.5 \mathrm{meV})$ and angular resolution $\left( \pm 0.7^{\circ}\right)$ but the most important difference is that the Scienta analyzer is able to measure the dispersion of the surface state over a wide range of angles simultaneously. In this way, all spectroscopic features of a phase transition can be detected, not merely a possible gap opening at the Fermi level crossing.

The data taken in Aarhus are shown in Fig. 4. The photoemission intensity at the Fermi level for a grid of $\mathbf{k}$ points close to $\bar{\Gamma}$ is presented in Fig. 4(a), revealing the hexagonal shape of the electron pocket. The figure shows raw data; no fitting was employed. Figure 4(b) shows a MDC taken at the Fermi level along $\bar{\Gamma}-\bar{K}$ together with a fit using a Lorentzian line and a linear background. The Lorentzian linewidth of $24 \mathrm{~m}^{-1}$ is slightly lower than the value of $32 \mathrm{~m}^{-1}$ reported in Ref. 9. It is not clear if this linewidth is the natural linewidth (as limited by electron-defect scattering) or if it is still limited by the resolution of our analyzer. Obviously, it is the angular resolution which would have most influence on the MDC linewidth. The effect of the energy resolution can be expected to be very small because of the steep dispersion.

From such data, we can infer the value of $q_{\mathrm{CDW}}$, i.e., the value of $2 k_{\mathrm{F}}$ in the $\bar{\Gamma}-\bar{K}$ direction. We obtain a value of $0.122(2) \AA^{-1}$. This is slightly bigger than the $q_{\mathrm{CDW}}$ value obtained by Ast and Höchst. As in the work of Ast and Höchst, the error bars reflect the statistical error from the fit, which is very small. The difference between the value reported here and that reported in Ref. 9 is probably due to a different value of the sample work function, used to calculated $\mathbf{k}$ values from the spectrometer angles. The work function value we have used is $4.10(15) \mathrm{eV}$. It was obtained by taking a large-scale Fermi surface map in which three $\bar{\Gamma}$ points can be observed [see the inset in Fig. 4(a)]. Since the $k$ values for all the $\bar{\Gamma}$ points are precisely known, the work function can be evaluated.

Figure 4(c) shows a series of energy distribution curves (EDCs) taken exactly at the Fermi level crossing of the electron pocket in the $\bar{\Gamma}-\bar{K}$ direction as a function of temperature. The EDCs consist of one clear peak and a shoulder. The peak at a binding energy of about $80 \mathrm{meV}$ stems from the surface 

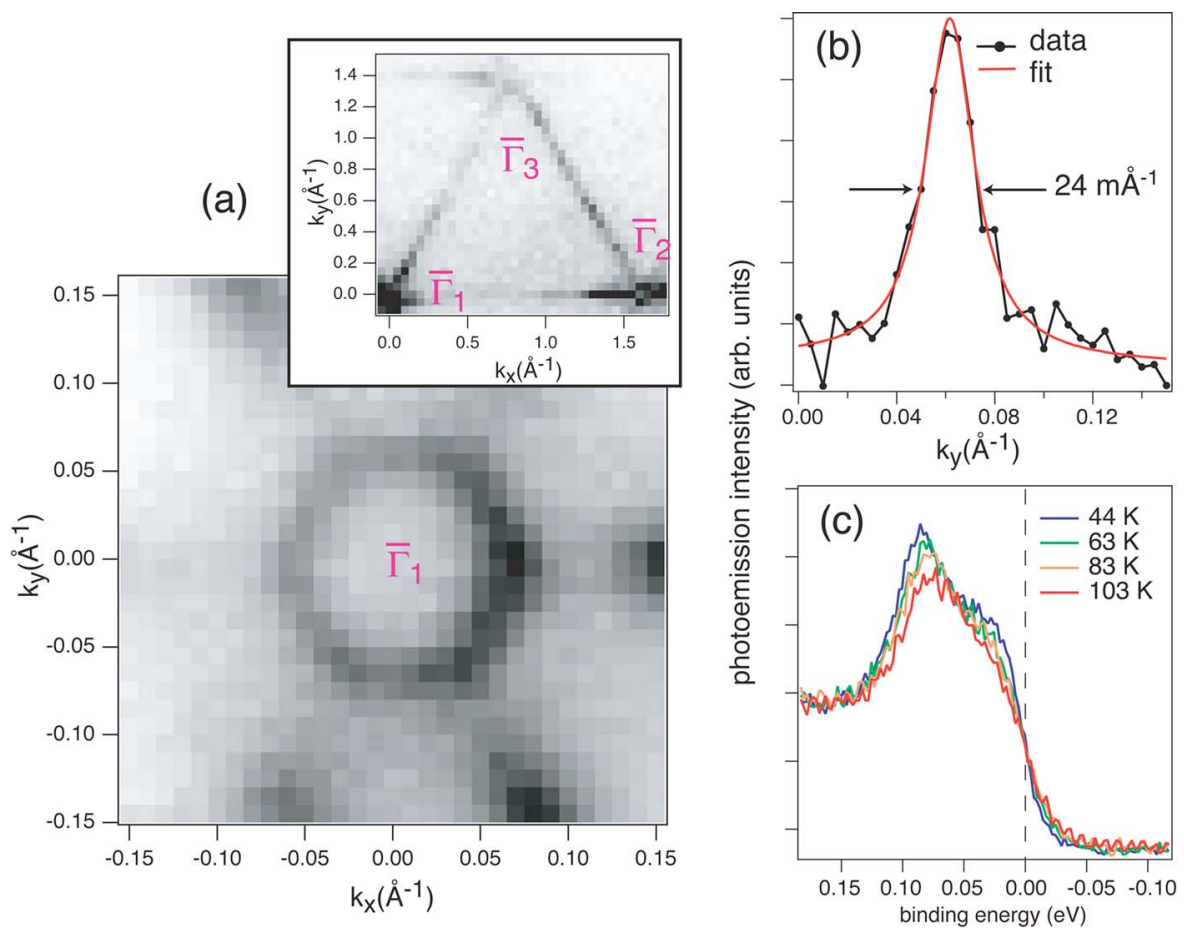

FIG. 4. (Color online) ARPES data taken in Aarhus. (a) Large image: photoemission intensity at the Fermi level (Fermi surface map) around normal emission, revealing the hexagonal shape of the electron pocket $(h \nu=15 \mathrm{eV}$ and $T=30 \mathrm{~K}$ ); smaller image: large scale Fermi surface map to determine sample orientation and work function $(h \nu=25 \mathrm{eV} \quad$ and $T=30 \mathrm{~K}$ ). (b) MDC taken at the Fermi level for the crossing of the electron pocket in the $\bar{\Gamma}-\bar{K}$ direction together with a Lorentzian fit $(h \nu=15 \mathrm{eV}$ and $T=30 \mathrm{~K})$. (c) EDCs taken at the Fermi level crossing in the $\bar{\Gamma}-\bar{K}$ direction as a function of temperature $(h \nu$ $=15 \mathrm{eV}$ ).

state band forming the hole pocket. The shoulder close to the Fermi level corresponds to the electron pocket. It does not show up as a clear peak because its maximum lies at the Fermi level. As a consequence of this, the Fermi edge appears to be much broader than expected from the energy resolution, a fact which is well known from other studies. ${ }^{12,38}$ Most importantly, there is no sign of a leading-edge gap opening at the lowest temperatures.

Figure 5 shows the temperature-dependent data set taken in Dresden. The value of $q_{\mathrm{CDW}}$ obtained from these data is $0.121(1) \AA^{-1}$; the value of the work function is $4.09(15) \mathrm{eV}$. Both were obtained in the same way as the Aarhus data and are in good agreement with those. Also in this data set, we do

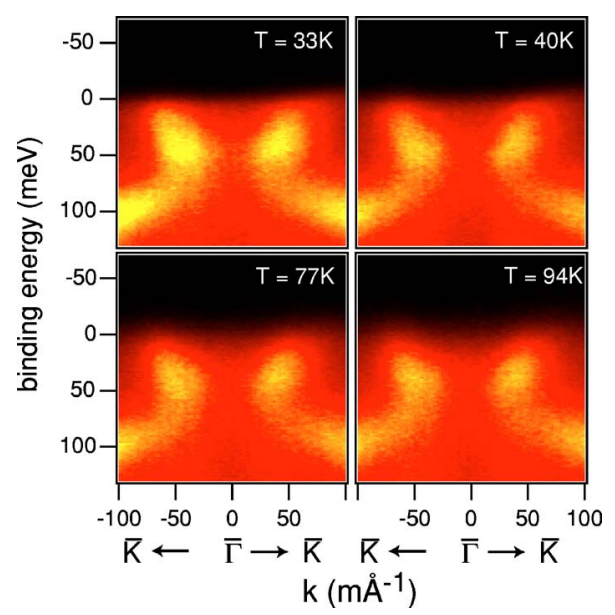

FIG. 5. (Color online) ARPES data taken in Dresden: Temperature-dependent dispersion of the surface states along $\bar{K}-\bar{\Gamma}-\bar{K}(h \nu=21.2 \mathrm{eV})$. The band crossing the Fermi level is the electron pocket. The band at a binding energy of about $100 \mathrm{meV}$ is the state which forms the hole pockets in the $\bar{\Gamma}-\bar{M}$ direction. not observe a gap opening at the Fermi level crossing. The band forming the electron pocket is somewhat broader than in the Aarhus data, most probably due to the lower angular resolution and the higher photon energy. Note, however, that a lower angular resolution would have no effect on the observation of an absolute band gap in the electronic structure.

Taking a large range of angles at one go has certain advantages over taking single EDCs in a selected $\mathbf{k}$ point. First of all, the experiment is much less sensitive towards changes of the sample alignment with temperature because the effect of such changes would be easily detected as a shift of the whole dispersion. In addition to this, one directly observes that there is no qualitative change in the dispersion which could hint towards a phase transition. It is well known, after all, that CDW-type transitions lead to a strong change of the dispersion close to the Fermi level (for a recent and rather dramatic example, see Ref. 39).

\section{CONCLUSION}

In conclusion, we do not find any indication for the formation of a charge-density wave on $\operatorname{Bi}(111)$ using STM and TEM. Our ARPES data confirm the hexagonal shape of the electron pocket around $\bar{\Gamma}$, which was described by Ast and Höchst, ${ }^{9}$ but we do not find an indication of a leading-edge gap opening in ARPES data from two different setups.

If we consider only the strong nesting in the hexagonal electron pocket, it is remarkable that $\mathrm{Bi}(111)$ does not have a CDW ground state, not even at the lowest temperatures of $5 \mathrm{~K}$ reached in the STM experiments. One important reason for this is probably the spin-orbit splitting in the surface state, which we have confirmed here experimentally using the phenomenon of quasiparticle interference in STM. It 
leads to the peculiar situation where $\mathrm{Bi}(111)$ and the other surfaces of Bi are good metals but with very unusual dynamical properties, for example, in the screening.

\section{ACKNOWLEDGMENTS}

We would like to thank G. Bihlmayer, S. Blügel, E. V. Chulkov, Y. M. Koroteev, and P. M. Echenique for many stimulating discussions in connection with this project and
Roland Hübel for technical support. This work was supported in part by the Danish National Science Foundation and by the U.S. Department of Energy under Contract No. DEFG0202ER45996. A part of the work was performed under the auspices of the U.S. Department of Energy by University of California Lawrence Livermore National Laboratory under Contract No. W-7405-Eng-48 and Lawrence Berkeley National Laboratory under Contract No. DE-AC0376-00098.
*Corresponding author. Electronic address: philip@phys.au.dk

${ }^{1}$ G. Grüner, Density Waves in Solids, Frontiers in Physics Vol. 89 (Perseus Publishing, Cambridge, MA, 1994).

${ }^{2}$ E. Tosatti, in Electronic Surface and Interface States on Metallic Systems, edited by E. Bertel and M. Donath (World Scientific, Singapore, 1995).

${ }^{3}$ T. Aruga, J. Phys.: Condens. Matter 14, 8393 (2002).

${ }^{4}$ E. Tosatti, Festkoerperprobleme XV, 113 (1975).

${ }^{5}$ S. Agergaard, C. Søndergaard, H. Li, M. B. Nielsen, S. V. Hoffmann, Z. Li, and Ph. Hofmann, New J. Phys. 3, 15.1 (2001).

${ }^{6}$ M. Hengsberger, P. Segovia, M. Garnier, D. Purdie, and Y. Baer, Eur. Phys. J. B 17, 603 (2000).

${ }^{7}$ C. R. Ast and H. Höchst, Phys. Rev. Lett. 87, 177602 (2001).

${ }^{8} \mathrm{Ph}$. Hofmann, J. E. Gayone, G. Bihlmayer, Y. M. Koroteev, and E. V. Chulkov, Phys. Rev. B 71, 195413 (2005).

${ }^{9}$ C. R. Ast and H. Höchst, Phys. Rev. Lett. 90, 016403 (2003).

${ }^{10}$ Y. M. Koroteev, G. Bihlmayer, J. E. Gayone, E. V. Chulkov, S. Blügel, P. M. Echenique, and Ph. Hofmann, Phys. Rev. Lett. 93, 046403 (2004).

${ }^{11}$ A. Damascelli, Z. Hussain, and Z.-X. Shen, Rev. Mod. Phys. 75, 473 (2003).

${ }^{12}$ A. A. Kordyuk, S. V. Borisenko, M. Knupfer, and J. Fink, Phys. Rev. B 67, 064504 (2003).

${ }^{13}$ F. Jona, Surf. Sci. 8, 57 (1967).

${ }^{14}$ H. Mönig, J. Sun, K. Pohl, J. Wells, and Ph. Hofmann, Phys. Rev. B 72, 085410 (2005).

${ }^{15}$ G. Jezequel, Y. Petroff, R. Pinchaux, and F. Yndurain, Phys. Rev. B 33, 4352 (1986).

${ }^{16}$ F. Patthey, W.-D. Schneider, and H. Micklitz, Phys. Rev. B 49, 11293 (1994).

${ }^{17}$ G. Jezequel, J. Thomas, and I. Pollini, Phys. Rev. B 56, 6620 (1997).

${ }^{18}$ J. Thomas, G. Jezequel, and I. Pollini, J. Phys.: Condens. Matter 11, 9571 (1999).

${ }^{19}$ C. R. Ast and H. Höchst, Phys. Rev. B 66, 125103 (2002).

${ }^{20}$ R. V. Coleman, B. Drake, P. K. Hansma, and G. Slough, Phys. Rev. Lett. 55, 394 (1985).

${ }^{21}$ W. Han, R. A. Pappas, E. R. Hunt, and R. F. Frindt, Phys. Rev. B
48, 8466 (1993).

22 J. M. Carpinelli, H. H. Weitering, E. W. Plummer, and R. Stumpf, Nature (London) 381, 398 (1996).

${ }^{23}$ I. Ekvall, J.-J. Kim, and H. Olin, Phys. Rev. B 55, 6758 (1997).

${ }^{24}$ V. S. Edel'man, Phys. Lett. A 210, 105 (1996).

${ }^{25}$ A. V. Ofitserov and V. S. Edelman, Physica B 329-333, 1094 (2003).

${ }^{26}$ J. I. Pascual, G. Bihlmayer, Y. M. Koroteev, H.-P. Rust, G. Ceballos, M. Hansmann, K. Horn, E. V. Chulkov, S. Blügel, P. M. Echenique, and Ph. Hofmann, Phys. Rev. Lett. 93, 196802 (2004).

${ }^{27}$ M. F. Crommie, C. P. Lutz, and M. Eigler, Nature (London) 363, 524 (1993).

${ }^{28}$ L. Petersen, P. T. Sprunger, Ph. Hofmann, E. Lægsgaard, B. G. Briner, M. Doering, H.-P. Rust, A. M. Bradshaw, F. Besenbacher, and E. W. Plummer, Phys. Rev. B 57, R6858 (1998).

${ }^{29}$ Ph. Hofmann, B. G. Briner, M. Doering, H.-P. Rust, E. W. Plummer, and A. M. Bradshaw, Phys. Rev. Lett. 79, 265 (1997).

${ }^{30}$ J. E. Hoffman, K. McElroy, D.-H. Lee, K. M. Lang, H. Eisaki, S. Uchida, and J. C. Davis, Science 297, 1148 (2002).

${ }^{31}$ K. McElroy, R. W. Simmonds, J. E. Hoffman, D.-H. Lee, J. Orenstein, H. Eisaki, and J. C. Davis, Nature (London) 422, 592 (2003).

${ }^{32}$ L. Diekhöner, M. A. Schneider, A. N. Baranov, V. S. Stepanyuk, P. Bruno, and K. Kern, Phys. Rev. Lett. 90, 236801 (2003).

${ }^{33}$ M. Vershinin, S. Misra, S. Ono, Y. Abe, Y. Ando, and A. Yazdani, Science 303, 1995 (2004).

${ }^{34}$ J. I. Pascual, Z. Song, J. J. Jackiw, K. Horn, and H.-P. Rust, Phys. Rev. B 63, 241103(R) (2001).

${ }^{35}$ P. T. Sprunger, L. Petersen, E. W. Plummer, E. Lægsgaard, and F. Besenbacher, Science 275, 1764 (1997).

${ }^{36}$ C. H. Chen and G. H. Lander, Phys. Rev. Lett. 57, 110 (1986).

${ }^{37}$ S. V. Hoffmann, C. Søndergaard, C. Schultz, Z. Li, and Ph. Hofmann, Nucl. Instrum. Methods Phys. Res. A 523, 441 (2004).

${ }^{38}$ J. Kröger, T. Greber, T. J. Kreutz, and J. Osterwalder, J. Electron Spectrosc. Relat. Phenom. 113, 241 (2001).

${ }^{39}$ S. Hatta, H. Okuyama, M. Nishijima, and T. Aruga, Phys. Rev. B 71, 041401(R) (2005). 